\title{
Neovascularization after ischemic conditioning of the stomach and the influence of follow-up neoadjuvant chemotherapy thereon
}

\author{
Vadim Prudius ${ }^{1}$, Vladimír Procházka ${ }^{2}$, Zdeněk Pavlovský ${ }^{3}$, Adam Peštál $^{1}$, Petr Vlček ${ }^{1}$, Ivan Čapov ${ }^{1}$, Lenka Veverková ${ }^{1}$, \\ Michal Reška ${ }^{1}$ \\ ${ }^{1} 1^{\text {st }}$ Surgical Department, Masaryk University and Saint Anne's University Hospital, Brno, Czech Republic \\ ${ }^{2}$ Surgical Clinic, University Hospital Brno, Brno, Czech Republic \\ ${ }^{3}$ Institute of Pathology, University Hospital Brno, Brno, Czech Republic
}

Videosurgery Miniinv 2018; 13 (3): 299-305

DOI: https://doi.org/10.5114/wiitm.2018.75907

\begin{abstract}
Introduction: Esophagectomy and reconstruction remain the optimal treatment for patients with resectable esophageal cancer. Neovascularization after ischemic conditioning of the stomach before esophagectomy is a laparoscopic procedure which may potentially reduce gastric conduit ischemia.

Aim: To investigate the influence of ischemic conditioning on neovascularization along the greater curvature of the stomach and to explore the effect of neoadjuvant chemotherapy on neovascularization after ischemic conditioning. Material and methods: Staging laparoscopy was performed before the main resection procedure; during this procedure ischemic conditioning was performed. Samples taken from the human stomach were divided into 3 groups: group A - patients after ischemic conditioning with a delay of 30-45 days after left gastric artery (LGA) ligation $(n=4)$; group $B$ - patients who were undergoing neoadjuvant chemotherapy with a delay of 90-140 days after left gastric artery ligation $(n=4)$; and control group $C$ - patients without ischemic conditioning $(n=7)$.

Results: After ischemic conditioning with a delay of 30-45 days, the count of neovessels along the greater curvature of the stomach increased from $5.4 \pm 0.7$ in the control group to $17.5 \pm 0.9$ in a low-power field of view (LPF) in group $A$ and increased still further on average to $19.8 \pm 10.4$ in group $B$.

Conclusions: Left gastric artery ligation only is a sufficient procedure for ischemic conditioning of the stomach. Neovascularization along the greater curvature is a continuous process that depends on delay time. Neoadjuvant therapy has no influence on the effect of neovascularization.
\end{abstract}

Key words: esophageal cancer, neovascularization, esophageal surgery, gastric conduit, ischemic conditioning.

\section{Introduction}

The incidence of esophageal cancer is increasing year by year. The only curative method of treatment in this area remains surgical resection. This operation is associated with a high incidence of postoperative leak in the anastomosis (around 20\%) and a mortality rate of around 3-5\% [1-3]. The length of survival in the postoperative period is not the sole criterion of surgical effectiveness. The quality of life of our patients also plays a large role in their level of satisfaction.

The most widely used surgical operation for this condition is esophageal resection with gastric pull-up replacement. Up to $50 \%$ of patients who have postoperative leak during esophagogastroanastomosis are faced with the need for dilation of the anastomosis due to stenosis $[4,5]$. One of the most important 
causes of this problem is a lack of blood supply in the stomach conduit [6]. Basically, the only source of gastric conduit blood supply remains the right gastroepiploic artery (RGEA), also known as the right gastro-omental artery [7, 8]. Ischemic conditioning of the stomach occurring after left gastric artery (LGA) discontinuation is a procedure used in a number of surgical departments around the world. This is a completely laparoscopic procedure with zero percent mortality and almost zero morbidity. This procedure may potentially reduce leakage after esophagogastroanastomosis construction. The optimal timing of the procedure remains questionable. The influence of neoadjuvant therapy and the sheer number of vessels that need to be interrupted for the fully-fledged effect of ischemic conditioning to arise are unknown. There are a number of studies demonstrating the effect of conditioning on animal models [9-11].

\section{Aim}

There are a number of issues addressed by our study: whether ligation of the LGA alone is sufficient intervention to produce the effect of ischemic conditioning and initiate neovascularization of the stomach wall, whether neoadjuvant therapy can affect neovascularization after ischemic conditioning, and whether neovascularization after ischemic conditioning is a time-dependent process.

\section{Material and methods}

We conducted a prospective study. Patients with a resectable or potentially resectable tumor of the middle and aboral esophagus were included. Staging laparoscopy was performed before the main resection procedure. The laparoscopic procedure is performed standardly on the patient's back. Three abdominal ports (one $5 \mathrm{~mm}$, and two $11 \mathrm{~mm}$ ) are used for the dissection. The gastrohepatic ligament is divided. After the LGA is found, it is clipped with two self-closing plastic endoscopic clips and cut between the clips. Drains for this procedure are not used. There were no conversions to an open procedure. Length of stay was $3 \pm 2$ days. We did not have any postoperative complications after the ischemic conditioning procedure. This study did not include patients in whom conditioning was combined with jejunostomy. No death after ischemic conditioning occurred.

After the main resection procedure (esophagectomy), samples of the resected stomach wall were obtained with a minimum distance of $5 \mathrm{~cm}$ from the macroscopic border of the tumor. This study focused primarily on the effect of ischemic conditioning on neovascularization of the stomach.

The patients in the main group were divided into two subgroups. Group A comprised patients with primary resectable tumors of the esophagus - a total of 4 patients. Resection surgery was performed 30-45 days after LGA ligation.

Group B comprised patients with potentially resectable tumors - a total of 4 patients. They underwent neoadjuvant chemotherapy (5-fluorouracil plus cisplatin). Restaging and the main surgical procedure were conducted in the period from 90 to 140 days and we recorded a downsizing of the tumor in all cases. All were evaluated as resectable and a curative resection was performed. Metastasis was not recorded during chemotherapy.

Resected stomachs in patients after laparoscopically performed sleeve gastrectomy were allocated to group C (control). All histological material obtained from bariatric patients was routinely examined histologically. These were healthy stomachs without any macroscopic or microscopic pathology and without the presence of polyps or gastric ulcers. In relation to comorbidities, we tolerated only compensated non-insulin-dependent diabetes mellitus type II with $\mathrm{HbA}_{1 \mathrm{c}}<60 \mathrm{mmol} / \mathrm{l}$ and a mild degree of bronchial asthma. These patients did not use any drugs affecting either arterial pressure or venotonics. They did not use hormones or proton pump inhibitors. In one case, we managed to obtain material from a patient with a tumor of the distal third of the esophagus who had not undergone staging laparoscopy with ligation of the LGA. This sample was added to the control group.

\section{Material sampling}

In all cases, standard samples were taken from resected stomachs. We took samples along the stapler line in the fundus and the body of the stomach. The distance from the greater curve was approximately $45 \mathrm{~mm}$. The upper area of the samples matched the area of future anastomosis of the gastric pull-up. Samples from a total of 15 patients were used.

\section{Processing of materials}

Stomachs were examined in the standard way after resection to rule out the presence of malignant 
or other pathological lesions in the tissue specimen. Tissue samples were taken from the same fully compatible location in the case of both stomach conduit construction and bariatric surgery. Tissue samples were fixed in $4 \%$ paraformaldehyde with phosphate buffer, $\mathrm{pH} 7.4$ at $18^{\circ} \mathrm{C}$. After fixation, the tissue was embedded in paraffin and cut into 7-10 $\mu \mathrm{m}$ sections for immunohistochemical and histological examination. A histopathological tissue survey was generally conducted after routine staining with hematoxylin and eosin. Neovessels were evaluated using immunohistochemical verification with CD31 (cluster of differentiation 31) and smooth muscle actin detection. Samples were examined under $80 \times$ magnification - low power field (LPF) and evaluation of the number of vessels was performed recalculated to one field of view. Ten fields of view were evaluated for each specimen.

After histopathological and immunohistochemical results were obtained, the material was coded by an independent collaborator (supervisor) and a randomized, blind outcome assessment subsequently conducted. Double-blind, randomized summation was then performed. We preferred evaluation of blood vessels in the areas of their greatest expression in the submucosal layer. No blood vessel was found in only two cases in group $C$ at 1 LPF at $80 \times$ magnification. After the results were submitted they were arranged in Microsoft Excel spreadsheets.

After admission, all the patients gave their consent in writing to hospitalization and to diagnostic and therapeutic acts connected with this hospitalization. They thereby expressed their will to decide freely about procedures when accepting healthcare services. They are entitled to ask supplementary questions to find out about anything they consider important and all their queries are answered by the physician. They sign a declaration confirming that they find the information and explanations given to them fully satisfactory and that they understand everything and agree to the healthcare provided during hospitalization. This informed consent corresponds to Act No. 372/2011 on conditions of healthcare services in the Czech Republic (CR).

The Ethics Committee issued a favorable opinion of research project FW03/15 (Neovascularization after ischemic conditioning and the influence of follow-up neoadjuvant chemotherapy thereon) at Saint Anne's Faculty Hospital.

\section{Results}

The results were divided into the pertinent groups. Group A comprised patients with tumors of the esophagus at least $5 \mathrm{~cm}$ from the site of histological sampling. The patients in this group did not have neoadjuvant therapy and underwent a primary resection procedure between 30 and 45 days after staging laparoscopy with LGA ligation - a total of 4 patients. Group B comprised patients who had neoadjuvant chemotherapy and underwent a resection procedure 90-140 days after staging laparoscopy (restaging was performed in all patients) - a total of 4 patients. Group C was the control group: patients after sleeve gastrectomy with an intact stomach wall - a total of 6 patients plus one histological sample (\#14) from a patient who had not undergone staging laparoscopy with LGA ligation. This patient had a primary resection procedure. The complete results are shown in Table I.

In group $\mathrm{A}$, we recorded a three-fold increase in the number of vessels with maximum expression in the submucosal layer [12]. An average of 17.47 vessels were found in each LPF. The diameter of vessels was not dissimilar to the diameter of vessels in

Table I. Description of samples

\begin{tabular}{|lccc|}
\hline Sample & Group & $\begin{array}{c}\text { Conditioning } \\
\text { time }\end{array}$ & $\begin{array}{c}\text { Vessels on } \\
\text { LPF }\end{array}$ \\
\hline 1 & A & 45 days & 17.57 \\
\hline 2 & A & 41 days & 16.57 \\
\hline 3 & A & 36 days & 17.71 \\
\hline 4 & A & 36 days & 18.25 \\
\hline 5 & B & 97 days & 12.0 \\
\hline 6 & B & 140 days & 32.75 \\
\hline 7 & B & 120 days & 21.87 \\
\hline 8 & B & 133 days & 12.75 \\
\hline 9 & C & 0 & 5.87 \\
\hline 10 & C & 0 & 4.62 \\
\hline 11 & C & 0 & 4.87 \\
\hline 12 & C & 0 & 5.12 \\
\hline 13 & C & 0 & 4.87 \\
\hline 14 & C & 0 & 6.0 \\
\hline 15 & C & 0 & 6.64 \\
\hline
\end{tabular}



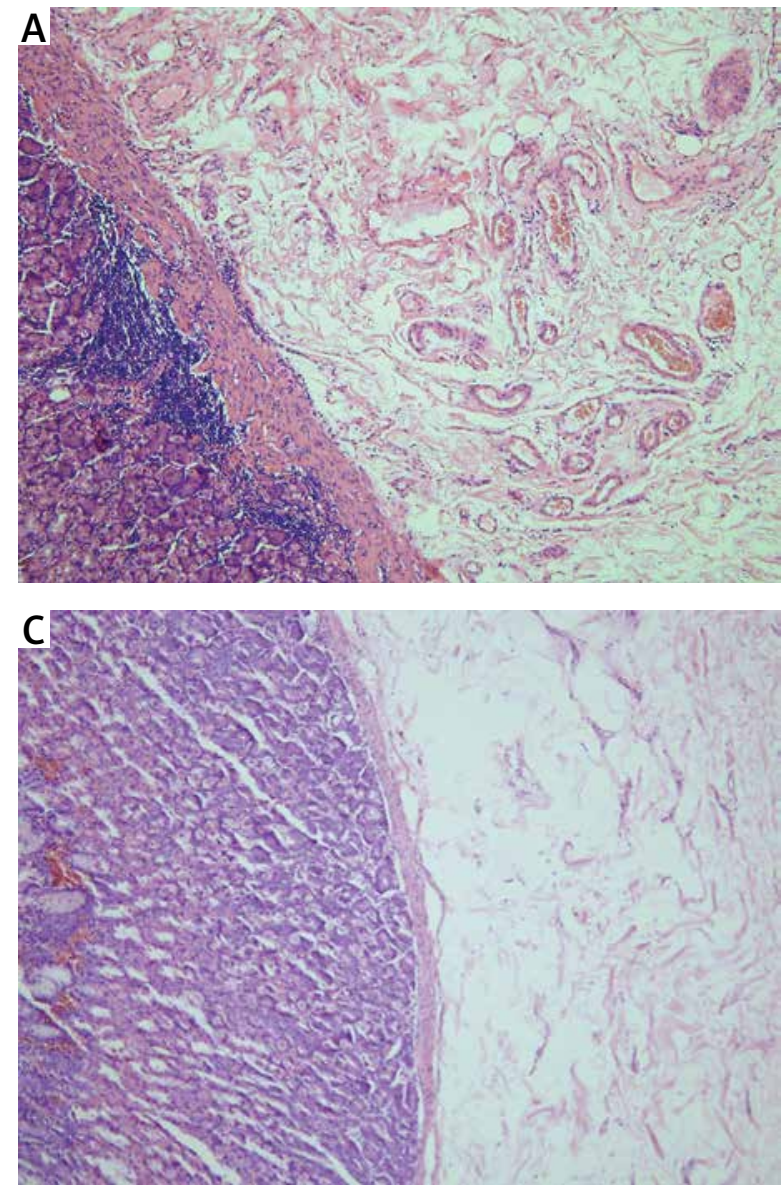

the samples in group C. No significant differences in the number of vessels on average in each LPF were observed between the samples. Sample $1-17.57$ vessels, sample $2-16.37$, sample $3-17.71$, sample 4-18.25 (Photo 1 A).

A larger number of blood vessels was recorded in all samples in group B as compared to the control group. The acceleration of vascular growth was not, however, homogeneous. In 2 samples after 140 and 120 days we found 32.75 and 21.87 vessels on one LPF. Vessel diameter was the same as in group A. In two other samples with a delay of 97 and 133 days we found an average of 12.0 and 12.75 vessels on one LPF. A twofold or threefold increase in vessel diameter in comparison with group A was also recorded (Photo $1 \mathrm{~B}$ ).

There were seven samples in group $C$. The average number of vessels was 6 per field of view (Photo $1 \mathrm{C})$. Sample 14 had the same morphology as the other samples taken from bariatric patients. The results of the randomized vessel count are shown in Figures 1 and 2 .

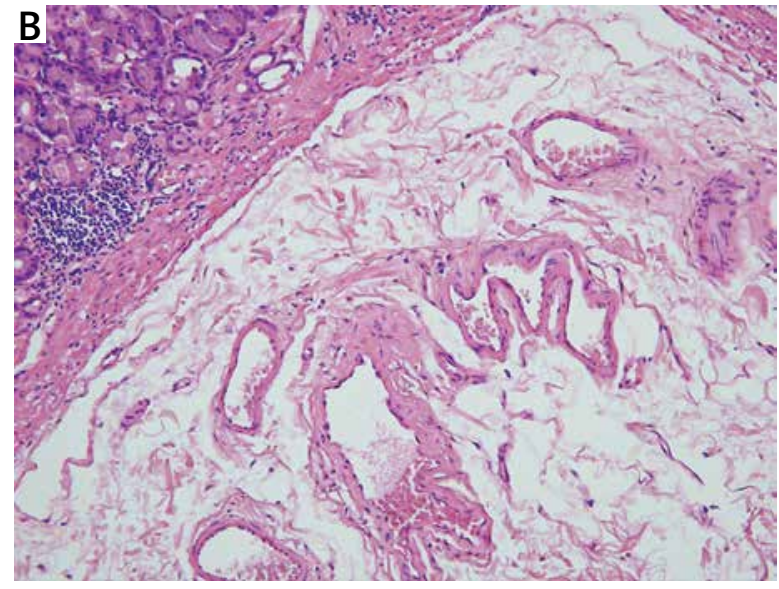

Photo 1. Histological slides of the stomach wall. A - After conditioning with a delay of 30-45 days. B - after conditioning with a delay of 90140 days. $\mathbf{C}$ - control group

These results show that neovascularization can probably occur in two ways - either by means of a further increase in the numbers of neovessels or their partial reduction with an increase in their caliber to preserve the increased flow through the vessels along the large curvature of the stomach.

\section{Discussion}

Laparoscopic ischemic conditioning of the stomach for esophageal replacement is a safe procedure [10]. During the course of laparoscopy, it is possible to perform not only an LGA discontinuation. In some cases, it is possible to detect a higher stage of the cancer than has been detected by computed tomography and endoscopic methods.

This study shows that ischemic conditioning of the stomach based on the ischemic stimulation of neovascularization is carried out according to the theoretical background and experimental results in animal models [9]. A number of studies have demon- 


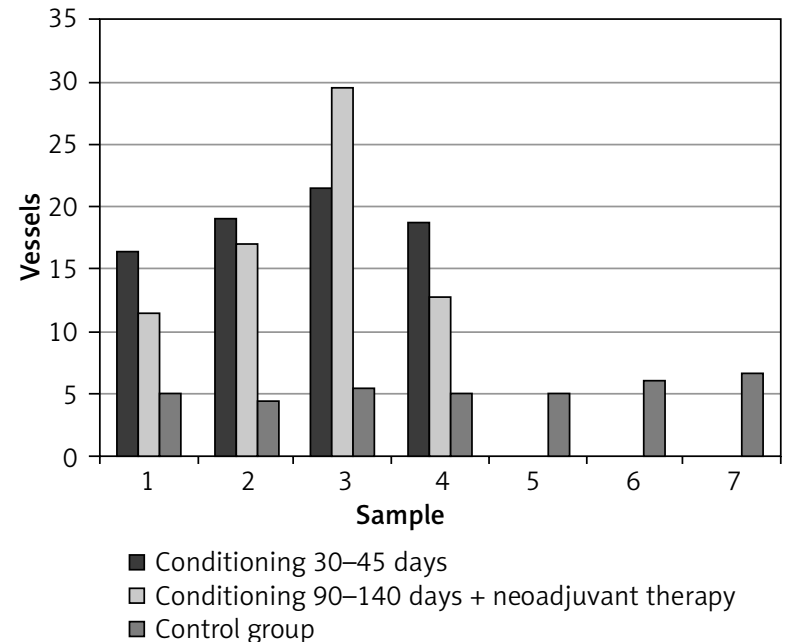

Figure 1. Comparison of the number of vessels in each sample

strated neovascularization in the stomach wall after ischemic conditioning [9, 13-15]. The construction of a gastric pull-up requires discontinuation of at least three of the five main blood vessels that provide stomach vascularity $[6,13]$. The only significant source of blood supply to the stomach remains the right gastroepiploic artery $[8,13,16,17]$. The right gastric artery is of only little significance [18]. In view of the fact that the RGEA supplies on average $50 \%$ of the length of the greater curve [7], the oral part of the conduit is supplied by an anastomosis between the vessels inside the stomach wall or by capillaries only [7]. The effect of ischemic conditioning aimed at accelerating growth in the number of blood vessels in the stomach wall is used to improve blood supply to the conduit. There is no consensus in the literature regarding the timing of stomach conditioning, but in recent times the delay has shifted from 2-5 days [19] up to 30 days $[10,11]$ or, in some reports, up to 75 days [8]. In spite of this change, the optimal timing remains debatable [20]. To date, only animal models directly reveal the effect of conditioning, and no studies have succeeded in demonstrating this effect directly in humans. We assume that the effect of neovascularization in a quantitative ratio in humans is different from that in animals. We have not found any literature suggesting the way in which neoadjuvant therapy influences the effect of ischemic conditioning.

The question of the influence of ischemic conditioning (IC) of the stomach on the incidence of

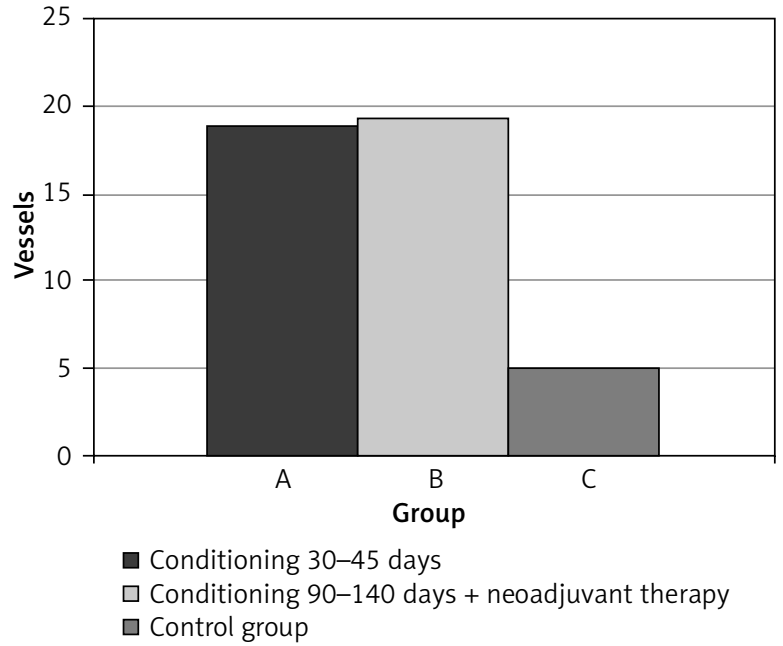

Figure 2. Summary of the number of vessels in each group

postoperative leakage remains highly controversial. Studies suggest that the incidence of leakage is not significantly altered in patients who undergo IC as compared to those who do not $[8,21,22]$, though some prove the positive effect of IC [19, 23]. Staging laparoscopy before esophagectomy has greater benefits than IC after LGA interruption. Staging laparoscopy can uncover undiagnosed locally advanced lymph node involvement in the abdominal part of the esophagus. This operation should not, however, be performed routinely [24, 25].

Significant neovascularization was found in groups $A$ and $B$ in comparison with the control group. The results of neovascularization in group $\mathrm{A}$ were well balanced. No significant changes in the average number of vessels on one LPF were found. The results were not homogeneous in group B, with a delay of over 90 days. We observed two tendencies: either a further increase in the number of blood vessels, maintaining the same average diameter, or a reduction in their number, though accompanied by an increase in their size (diameter). We cannot give a clear explanation of the discrepancy in the progression of vascular diameter for individual samples due to the small number of samples to date. In spite of the fact that the overall results in group $B$ were not homogeneous, the number of vessels being 19.8 \pm 10.4 to one LPF, this deviation is reduced when we assess the results in two subgroups. Subgroup B1, with an overall slightly lower number of neovessels but an increase in their diameter $(12.37 \pm 0.4$ to one 
LPF), and subgroup B2, with a continued increase in the number of vessels of the same vessel diameter $(26.9 \pm 5.3$ to one LPF).

Only LGA ligation was performed on all the patients within the framework of conditioning. This effect has been described in animal models [9, 1315]. It was found that hypoxia stimulates the production of angiogenic factors including platelet-derived growth factor (VEGF) [12]. There are several methods of ischemic conditioning of the stomach: embolization or ligation of vessels [20]. In our case, we achieved conditioning using the least traumatic method of LGA ligation to minimize adhesions during resectioning. Even though the blood flow through other arteries feeding the stomach was preserved, the effect of ischemic conditioning caused a multiple increase in the number of neovessels. This means that LGA ligation is a procedure that suffices to produce the effect of ischemic conditioning.

An increase in the number of vessels in comparison with group $A$ was recorded in evaluating the outcome in group B. Changes arising in subgroups B1 and B2 were due to the time shift rather than the influence of neoadjuvant therapy. Evidence for this is provided by the continuing increase in the number of vessels in subgroup B2.

\section{Conclusions}

Ligation of the LGA for the purpose of ischemic conditioning of the stomach before a resection procedure on the esophagus is a procedure that suffices to produce the effect of ischemic conditioning (neovascularization). An almost threefold acceleration in the growth of blood vessels can be seen. Neoadjuvant chemotherapy (assuming the use of 5-FU and cisplatin) does not affect neovascularization. The effect of ischemic conditioning was proven in all samples. Hypervascularization of the wall of the stomach continues even when the operation is delayed for up to 140 days after LGA ligation. The results obtained require further research into the effect of ischemic conditioning in general and neovascularization after ischemic conditioning of the stomach in particular. Given the small number of samples, it is difficult to rule out errors in the statistics. This study requires further investigation of the above-mentioned problem.

\section{Conflict of interest}

The authors declare no conflict of interest.

\section{References}

1. Cassivi SD Leaks, strictures, and necrosis: a review of anastomotic complications following esophagectomy. Semin Thorac Cardiovasc Surg 2004; 16: 124-32.

2. Kassis ES, Kosinski AS, Ross P Jr, et al. Predictors of anastomotic leak after esophagectomy: an analysis of the Society of Thoracic Surgeons General Thoracic database. Ann Thorac Surg 2013; 96: 1919-26.

3. Kruszewski WJ. Endoscopic methods in the treatment of early-stage esophageal cancer. Videosurgery Miniinv 2014; 9: 125-30.

4. Vrba R, Aujeský R, Vomáčková K, et al. Minimally invasive esophagectomy for esophageal cancer - results of surgical therapy. Videosurgery Miniinv 2015; 10: 189-96.

5. ReavisKM, Chang EY, Hunter JG, Jobe BA. Utilization of the delay phenomenon improves blood flow and reduces collagen deposition in esophagogastric anastomoses. Ann Surg 2005; 241: 736-45.

6. Boyle NH, Pearce A, Hunter D, et al. Intraoperative scanning laser Doppler flowmetry in the assessment of gastric tube perfusion during esophageal resection. J Am Coll Surg 1999; 188: 498-502.

7. Liebermann DM, Meier R, Siewert JR. Vascular anatomy of the gastric tube used for esophageal reconstruction. Ann Thorac Surg 1992; 54: 1110-5.

8. Nguyen NT, Nguyen XM, Reavis KM, et al. Minimally invasive esophagectomy with and without gastric ischemic conditioning. Surg Endosc 2012; 26: 1637-41.

9. PerryKA, Banarjee A, Liu J, et al. Gastric ischemic conditioning increases neovascularization and reduces inflammation and fibrosis during gastroesophageal anastomotic healing. Surg Endosc 2013; 27: 753-60.

10. Hölscher AH, Schneider PM, Gutschow C, Schröder W. Laparoscopic ischemic conditioning of the stomach for esophageal replacement. Ann Surg 2007; 245: 241-6.

11. Urschel JD, Antkowiak JG, Delacure MD, Takita H. Ischemic conditioning (delay phenomenon) improves esophagogastric anastomotic wound healing in the rat. J Surg Oncol 1997; 66: 254-6.

12. Schilling MK, Redaelli C, Maurer C, et al. Gastric microcirculatory changes during gastric tube formation: assessment with laser Doppler flowmetry. J Surg Res 1996; 62: 125-9.

13. Mittermair C, Klaus A, Scheidl S, et al. Functional capillary density in ischemic conditioning: implications for esophageal resection with the gastric conduit. Am J Surg 2008; 196: 88-92.

14. Akiyama S, Kodera Y, Sekiguchi H, et al. Preoperative embolization therapy for esophageal operation. J Surg Oncol 1998; 69: 219-23.

15. Nguyen NT, Longoria M, Sabio A, et al. Preoperative laparoscopic ligation of the left gastric vessels in preparation for esophagectomy. Ann Thorac Surg 2006; 81: 2318-20.

16. Ndoye JM, Dia A, Ndiaye A, et al. Arteriography of three models of gastric oesophagoplasty: the whole stomach, a wide gastric tube and a narrow gastric tube. Surg Radiol Anat 2006; 28: 429-37. 
17. Prudius V, Procházka V, Pavlovský Z, et al. Vascular anatomy of the stomach related to resection procedures strategy. Surg Radiol Anat 2017; 39: 433-40.

18. Matsuki M, Tanikake M, Kani H, et al. Dual-phase 3D CT angiography during a single breath-hold using 16- MDCT: assessment of vascular anatomy before laparoscopic gastrectomy. AJR Am J Roentgenol 2006; 186: 1079-85.

19. Schröder W, Stippel D, Gutschow C, et al. Postoperative recovery of microcirculation after gastric tube formation. Langenbecks Arch Surg 2004; 389: 267-71.

20. Lineaweaver WC, Lei MP, Mustain W, et al. Vascular endothelium growth factor, surgical delay, and skin flap survival. Ann Surg 2004; 239: 866-73.

21. Berrisford RG, Veeramootoo D, Parameswaran R, et al. Laparoscopic ischaemic conditioning of the stomach may reduce gastric-conduit morbidity following total minimally invasive oesophagectomy. Eur J Cardiothorac Surg 2009; 36: 888-93.

22. Veeramootoo D, Shore AC, Shields B, et al. Ischemic conditioning shows a time-dependent influence on the fate of the gastric conduit after minimally invasive esophagectomy. Surg Endosc 2010; 24: 1126-31.

23. Veeramootoo D, Shore AC, Wajed SA. Randomized controlled trial of laparoscopic gastric ischemic conditioning prior to minimally invasive esophagectomy, the LOGIC trial. Surg Endosc 2012; 26: 1822-9.

24. Krasna MJ, Flowers JL, Attar S, McLaughlin J. Combined thoracoscopic/laparoscopic staging of esophageal cancer. J Thorac Cardiovasc Surg 1996; 111: 800-6.

25. Berry MF. Esophageal cancer: staging system and guidelines for staging and treatment. J Thorac Dis 2014; 6 (Suppl 3): S289-97.

Received: 20.12.2017, accepted: 26.02.2018. 